\title{
Lipoenxertia e cirurgia plástica reconstrutiva da cicatriz cirúrgica
}

Fat grafting and reconstructive plastic surgery of the surgical scar

\section{Marcelo Moraes Valença}

Universidade Federal de Pernambuco, Recife, Pernambuco, Brasil

Universidade Federal de Pernambuco,

Recife, Pernambuco, Brasil

mmvalenca@yahoo.com.br

\begin{abstract}
Artigo publicado no Aesthetic Surgery Journal' avaliou alterações histológicas da lipoenxertia em um ensaio clínico de lipoenxertia-abdominoplastia em mulheres com índice de massa corporal $<30 \mathrm{~kg} / \mathrm{m}^{2}$ e com cicatriz de cesárea prévia. No estudo, essas mulheres foram submetidas a lipoenxertia seguida de abdominoplastia. ${ }^{1}$
\end{abstract}

Enxerto de gordura foi implantado em um dos lados. Quatro meses depois foi realizada uma abdominoplastia e amostras do tecido adiposo foram coletadas para análise histológica. Verificou-se que todas as amostras lipoenxertadas apresentavam sinais de viabilidade, sem diferenças histológicas quando comparadas com tecido adiposo contralateral onde não recebeu enxertia, em relação ao infiltrado inflamatório da pele, espessura da derme, densidade vascular e quantidade de fibras elásticas.

A lipoenxertia é uma modalidade de procedimento que está cada vez mais sendo utilizada na cirurgia plástica reconstrutiva, por propriedades mecânicas e talvez nutritivas o enxerto de tecido adiposo adjacente ou na intimidade da cicatriz parece beneficiar positivamente a recuperação estética. 2

\section{Referências}

1. Maricevich J, Lima M, Maricevich AC, et al. Histological Evaluation of the Skin After Fat Grafting: A Blinded, Randomized, Controlled Clinical Study. Aesthet Surg J. 2020;40:NP388-NP393.

2. Fredman R, Katz AJ, Hultman CS. Fat Grafting for Burn, Traumatic, and Surgical Scars. Clin Plast Surg. 2017;44:781-791. 